\title{
Quantum Chaos, Dynamical Stability and Decoherence
}

\author{
Giulio Casati ${ }^{1,2,3}$ and Tomaž Prosen ${ }^{4}$ \\ 1 Center for Nonlinear and Complex Systems, \\ Universita' degli Studi dell'Insubria, 22100 Como, Italy \\ ${ }^{2}$ Istituto Nazionale per la Fisica della Materia, \\ unita' di Como, 22100 Como, Italy, \\ 3 Istituto Nazionale di Fisica Nucleare, \\ sezione di Milano, 20133 Milano, Italy, \\ ${ }^{4}$ Department of Physics, Faculty of mathematics and physics, \\ University of Ljubljana, 1000 Ljubljana, Slovenia,
}

Received on 20 February, 2005

\begin{abstract}
We discuss the stability of quantum motion under system's perturbations in the light of the corresponding classical behavior. In particular we focus our attention on the so called "fidelity" or Loschmidt echo, its relation with the decay of correlations, and discuss the quantum-classical correspondence. We then report on the numerical simulation of the double-slit experiment, where the initial wave-packet is bounded inside a billiard domain with perfectly reflecting walls. If the shape of the billiard is such that the classical ray dynamics is regular, we obtain interference fringes whose visibility can be controlled by changing the parameters of the initial state. However, if we modify the shape of the billiard thus rendering classical (ray) dynamics fully chaotic, the interference fringes disappear and the intensity on the screen becomes the (classical) sum of intensities for the two corresponding one-slit experiments. Thus we show a clear and fundamental example in which transition to chaotic motion in a deterministic classical system, in absence of any external noise, leads to a profound modification in the quantum behavior.
\end{abstract}

\section{INTRODUCTION}

As it is now widely recognized, classical dynamical chaos has been one of the major scientific breakthroughs of the past century. On the other hand, the manifestations of chaotic motion in quantum mechanics, though widely studied $[1,2]$, remain somehow not so clearly understood, both from the mathematical as well as from the physical point of view.

The difficulty in understanding chaotic motion in terms of quantum mechanics is rooted in two basic properties of quantum dynamics:

(1) The energy spectrum of bounded, finite number of particles, conservative quantum systems is discrete. This means that the quantum motion is ultimately quasi-periodic, i.e. any temporal behavior is a discrete superposition of finitely or countably many Fourier components with discrete frequencies. In the ergodic theory of classical dynamical systems, such a quasi-periodic dynamics corresponds to the limiting case of integrable or ordered motion while chaotic motion requires continuous Fourier spectrum [3].

(2) Quantum motion is dynamically stable, i.e. initial errors propagate only linearly with time [4]. Linear instability is a typical feature of classical integrable systems and this contrasts the exponential instability which characterizes classical chaotic systems.

Therefore it appears that quantum motion always exhibits the characteristic features of classically integrable, regular motion which is just the opposite of dynamical chaos. However, it has been shown that this apparently paradoxical situation can be resolved with the introduction of different time scales inside which the typical features of classical chaos are present in the quantum motion also. Since these time scales diverge as Planck constant $\hbar$ goes to zero, no contradiction arises with the correspondence principle [5].

It has been remarked that, while exponential separation of orbits starting from slightly different initial conditions is associated to classical chaos, the situation in quantum mechanics is drastically different. Indeed the scalar product of two states $\left\langle\psi_{1} \mid \psi_{2}\right\rangle$ is time-independent due to unitarity of time evolution. This has led to the introduction of fidelity as a measure of stability of quantum motion [6]. More precisely one considers the overlap of two states which, starting from the same initial conditions, evolve under two slightly different Hamiltonians $H$ and $H_{\varepsilon}=H+\varepsilon V$. The fidelity is then given by $f(t)=\left|\left\langle\psi\left|\exp \left(\mathrm{i} H_{\varepsilon} t / \hbar\right) \exp (-\mathrm{i} H t / \hbar)\right| \psi\right\rangle\right|^{2}$. The quantity $f(t)$ can be seen as a measure of the so-called Loschmidt echo: a state $|\psi\rangle$ evolves for a time $t$ under the (unperturbed) Hamiltonian $H$, then the motion is reversed and evolves back for the same time $t$ under the (perturbed) Hamiltonian $H_{\varepsilon}$ and the overlap with the initial state $|\psi\rangle$ is considered.

However, we would like to stress that, in principle, such difference between classical and quantum mechanics actually does not exists. The Liouville equation, which describes classical evolution, is unitary and reversible as the Schrödinger equation. However, there exist time scales up to which quantum motion can share the properties of classical chaotic motion including the local exponential instability (see, e.g., Ref. [5]). Due to the existence of such time scales, what may be different, and indeed it is, is the degree of stability of dynamical motion. Indeed, as clearly illustrated in the analysis of the Loschmidt echoes with respect to variation of the wavefunction [4] or variation of the Hamiltonian [7], quantum motion turns out to be more stable than the classical motion.

The growing interest in quantum computers $[8,9]$ has attracted recent interest in this quantity as a measure of the stability of quantum computation in the presence of hardware imperfections or noisy gate operations. Confining ourselves 
to classically chaotic systems, the emerging picture which results from analytical and numerical investigations [7, 10-18] is that both exponential and Gaussian decays are present in the time behavior of fidelity. The strength of the perturbation determines which of the two regimes prevails. The decay rate in the exponential regime appears to be dominated either by the classical Lyapunov exponent or, according to Fermi golden rule, by the spreading width of the local density of states.

In addition, at least for short times, the decaying behavior depends on the initial state (coherent state, mixture, etc.). While it can be true that, for practical purposes, the short time behavior of fidelity may be the most interesting one, it is also true, without any doubt, that in order to have a clear theoretical understanding and identify a possible universal type of quantum decay one needs to consider the asymptotic behavior of fidelity. On the other hand, in the regime of very small perturbation, which may be of interest for practical quantum computation, one may in fact be interested also in the long-time behavior of fidelity in the so-called linear response regime.

This short review is composed of three parts. In section II we discuss the problem of classical fidelity, namely the stability of chaotic classical dynamics against external perturbations. We show quite clearly that the short time-decay of classical fidelity is governed by exponential instability (Lyapunov exponents), whereas the long-time decay is determined by the decay of correlations (Ruelle-Pollicott resonances). In Section III we discuss the fidelity decay of generic quantum systems. We discuss the correspondence with classical fidelity for short times and outline different regimes of decay with respect to the strength of perturbation. In Section IV we discuss a different, interesting connection between dynamical chaos and the quantum world, the so-called chaos induced decoherence. We show, by means of a simple numerical experiment the double-slit experiment, that classical chaos suppresses coherence and acts in a similar way as noise or external macroscopic number of freedoms which is usually invoked in order to explain decoherence.

\section{STABILITY OF CLASSICAL MOTION UNDER SYSTEM'S PERTURBATIONS}

In the paper [19], it has been shown that the asymptotic decay of classical fidelity for chaotic systems is not related to the Lyapunov exponent. Similarly to correlation functions, this decay can be either exponential or power law. In the former case, the decay rate is determined by the gap in the spectrum of the discretized Perron-Frobenius operator, in the latter case the power law has the same exponent as for correlation functions.

In order to illustrate the above results let us consider the classical fidelity $f(t)$ which can be defined as follows:

$$
f(t)=\int_{\Omega} \mathrm{d} \vec{x} \rho_{\varepsilon}(\vec{x}, t) \rho_{0}(\vec{x}, t),
$$

where the integral is extended over the phase space $\Omega$, and

$$
\rho_{0}(\vec{x}, t)=U_{0}^{t} \rho(\vec{x}, 0), \quad \rho_{\varepsilon}(\vec{x}, t)=U_{\varepsilon}^{t} \rho(\vec{x}, 0)
$$

give the (classical) evolution after $t$ steps (assuming that the time is discrete - measured in terms of an integer number $t$ of fundamental periods) of the initial density $\rho(\vec{x}, 0)$ (assumed to be normalized, i.e. $\left.\int d \vec{x} \rho^{2}(\vec{x}, 0)=1\right)$ as determined by the $t$-th iteration of the Perron-Frobenius operators $U_{0}$ and $U_{\varepsilon}$, corresponding to the Hamiltonians $H_{0}$ and $H_{\varepsilon}$, respectively. The above definition can be shown to correspond to the classical limit of quantum fidelity [7, 15]. For some other applications in the context of classical mechanics see Ref. [20]. In the ideal case of perfect echo $(\varepsilon=0)$, the fidelity does not decay, $f(t) \equiv 1$. However, due to chaotic dynamics, when $\varepsilon \neq 0$ the classical echo decay sets in after a time scale

$$
t_{\varepsilon} \sim \frac{1}{\lambda} \ln \left(\frac{v}{\varepsilon}\right),
$$

required to amplify the perturbation up to the size $v$ of the initial distribution. Thus, for $t \gg t_{\varepsilon}$ the recovery of the initial distribution via the imperfect time-reversal procedure fails.

Let us start by discussing the decay of classical fidelity in a standard model of classical chaos, characterized by uniform exponential instability, the so called sawtooth map.

The sawtooth map is defined by

$$
\bar{p}=p+F_{0}(\theta), \quad \bar{\theta}=\theta+\bar{p},
$$

where $(p, \theta)$ are conjugated action-angle variables, $F_{0}(\theta)=$ $K_{0}(\theta-\pi)$, and the overbars denote the variables after one map iteration. We consider this map on the torus $0 \leq \theta<2 \pi$, $-\pi L \leq p<\pi L$, where $L$ is an integer. For $K_{0}>0$ the motion is completely chaotic and diffusive, with Lyapunov exponent given by $\lambda=\ln \left\{\left(2+K_{0}+\left[\left(2+K_{0}\right)^{2}-4\right]^{1 / 2}\right) / 2\right\}$. For $K_{0}>1$ one can estimate the diffusion coefficient $D$ by means of the random phase approximation, obtaining $D \approx\left(\pi^{2} / 3\right) K_{0}^{2}$. In order to compute the fidelity (1), we choose to perturb the kicking strength $K=K_{0}+\varepsilon$, with $\varepsilon \ll K_{0}$. In practice, we follow the evolution of $10^{8}$ trajectories, which are uniformly distributed inside a given phase space region of area $A_{0}$ at time $t=0$. The fidelity $f(t)$ is given by the percentage of trajectories that return back to that region after $t$ iterations of the map (4) forward, followed by the backward evolution, now with the perturbed strength $K$, in the same time interval $t$. In order to study the approach to equilibrium for fidelity, we consider the quantity

$$
g(t)=(f(t)-f(\infty)) /(f(0)-f(\infty)) ;
$$

in this way $g(t)$ drops from 1 to 0 when $t$ goes from 0 to $\infty$. We note that $f(0)=1$ while, for a chaotic system, $f(\infty)$ is given by the ratio $A_{0} / A_{c}$, with $A_{c}$ being the area of the chaotic component to which the initial distribution belongs.

The behavior of $g(t)$ is shown in Fig. 1 , for $K_{0}=(\sqrt{5}+$ $1) / 2$ and different $L$ values. One can see that only the short time decay is determined by the Lyapunov exponent $\lambda, f(t)=$ $\exp (-\lambda t)$. In a recent paper [21] this short-time Lyapunov decay of classical fidelity has been explained theoretically, and an interesting cascade of decays with rates given by sums of largest Lyapunov exponents has been predicted and found for multi-dimensional systems. In our numerical example, the Lyapunov decay is followed by a power law decay [17] 


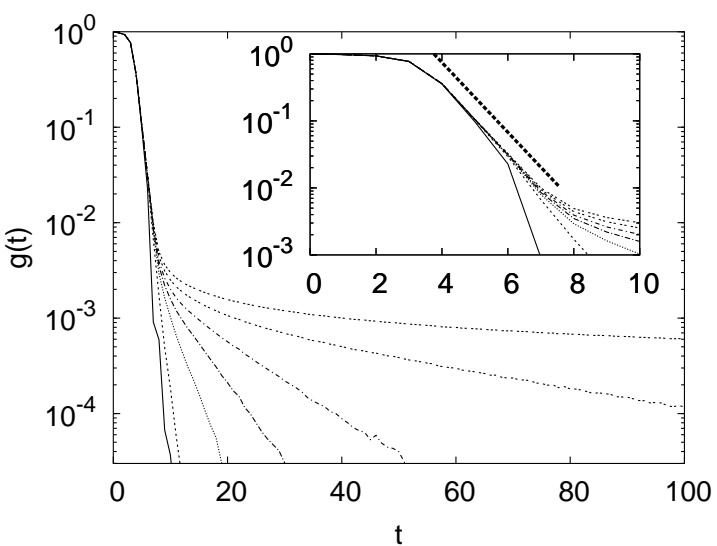

FIG. 1: Decay of the fidelity $g(t)$ for the sawtooth map with the parameters $K_{0}=(\sqrt{5}+1) / 2$ and $\varepsilon=10^{-3}$ for different values of $L=1,3,5,7,10,20, \infty$ from the fastest to the slowest decaying curve, respectively. The initial phase space density is chosen as the characteristic function on the support given by the $(q, p) \in[0,2 \pi) \times$ $[-\pi / 100, \pi / 100]$. Note that between the Lyapunov decay and the exponential asymptotic decay there is a $\propto 1 / \sqrt{t}$ decay, as expected from diffusive behavior. Inset: magnification of the same plot for short times, with the corresponding Lyapunov decay indicated as a thick dashed line.

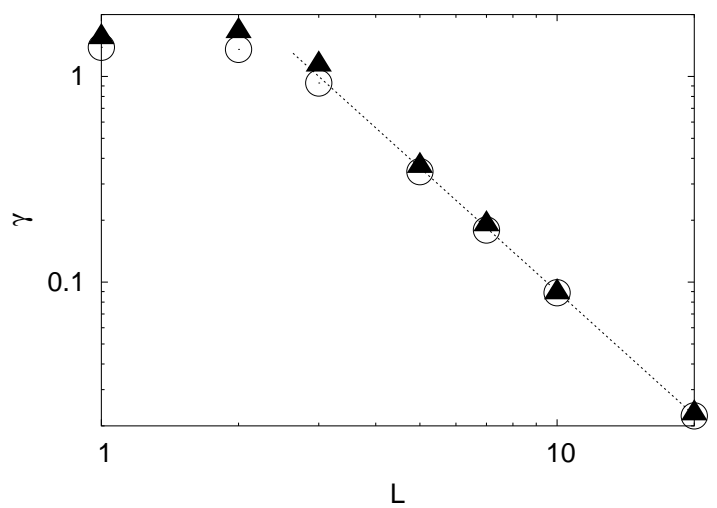

FIG. 2: Asymptotic exponential decay rates of fidelity for the sawtooth map $\left(K_{0}=(\sqrt{5}+1) / 2, \varepsilon=10^{-3}\right)$ as a function of $L$. The rates are extracted by fitting the tails of the fidelity decay in the Fig. 1 (triangles) and from the discretized Perron-Frobenius operator (circles). The line denotes the $\propto 1 / L^{2}$ behavior of the decay rates, as predicted by the Fokker-Planck equation.

$\propto 1 / \sqrt{D t}$ until the diffusion time $t_{D} \sim L^{2} / D$ and then the asymptotic relaxation to equilibrium takes place exponentially, with a decay rate $\gamma$ (shown in Fig. 2) ruled not by the Lyapunov exponent but by the largest Ruelle-Pollicott resonance [22].

We determine numerically these resonances for the sawtooth map using the method of Refs. [23, 24], namely by diagonalizing a discretized (coarse-grained) classical propagator [19].

In Fig. 2 we illustrate a good agreement between the asymptotic decay rate of fidelity (extracted from the data

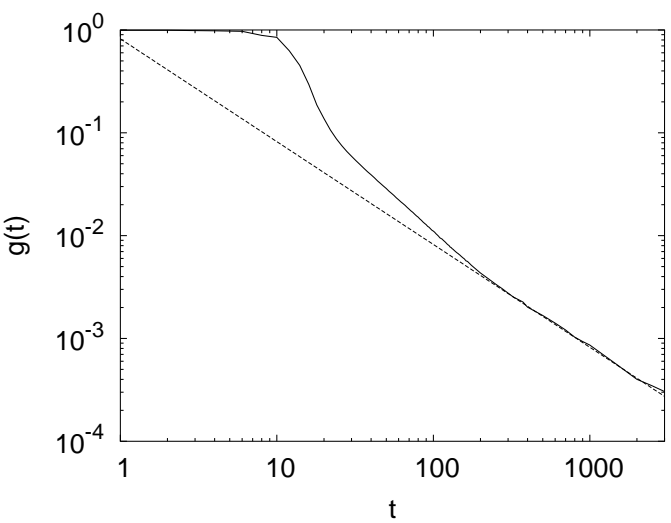

FIG. 3: Decay of fidelity for the stadium billiard with radius $R=1$ and length of the straight segments $d_{0}=2$ (the perturbed stadium has $d=d_{0}+\varepsilon$, with $\varepsilon=2 \times 10^{-3}$ ). The initial phase space density was chosen to be a direct product of a characteristic function on a circle in configuration space, the center of which was at $(0.5,0.25)$ as measured from the center of the billiard and its radius was 0.1 , while for momenta the $\delta(|\vec{p}|-1)$ distribution was used. The dashed line represents the expected $\propto 1 / t$ decay of fidelity.

of Fig. 1) and the decay rate $\gamma$ as predicted by the gap in the discretized Perron-Frobenius spectrum. We note that in the diffusive regime the classical motion can be described by the Fokker-Planck equation $(\partial / \partial t) R(p, t)=$ $(D / 2)\left(\partial^{2} / \partial p^{2}\right) R(p, t)$, where $R(p, t)=\int_{0}^{2 \pi} d \theta \rho(\theta, p, t)$ and $D \propto K_{0}^{2}$ is the diffusion coefficient. This gives an asymptotic relaxation rate $\gamma \propto K_{0}^{2} / L^{2}$, in agreement with the numerical data of Fig. 2. However, the argument based on the gap in the discretized Perron-Frobenius operator has a more general validity, and applies also in situations in which there is exponential relaxation but no diffusion, for example in the sawtooth map with $L=1$ (see Fig. 2).

We also point out that curves very similar to those plotted in Fig. 1 are obtained in the presence of stochastic noise, e.g. when the backward evolution is driven by a time-dependent kicking strength $K(t)=K_{0}+\varepsilon(t)$, with $\{\varepsilon(t)\}_{t=1,2, \ldots}$ uniformly and randomly distributed inside the interval $[-\varepsilon, \varepsilon]$. This means that the effect of a noisy environment on the decay of fidelity for a classically chaotic system is similar to that of a generic static Hamiltonian perturbation.

Further confirmation for the validity of the above illustrated scenario has been obtained by analyzing systems in which the initial phase space distribution is located inside a chaotic region and the asymptotic decay of correlations is algebraic. The latter decay can be due to the presence of arbitrarily long segments of regular motion in the time evolution of chaotic orbits [25] (like the billiard in a stadium) or to the sticking of trajectories in the vicinity of integrable components in systems with mixed phase space dynamics [26]. In the long time limit $\left(t \gg t_{\varepsilon}\right)$ the fidelity decay at time $t$ is still related to the decay of correlations at time $2 t$. Therefore, if correlations decay as $t^{-\alpha}$, fidelity decays with the same exponent $\alpha$. We have checked this expectation for the stadium billiard $(\alpha=1$ [25], see Fig. 3) and for the kicked rotator model (described by Eq. 


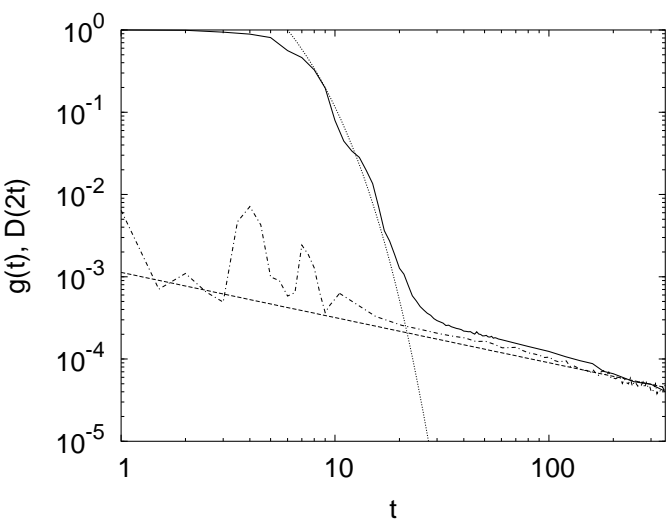

FIG. 4: Decay of fidelity for the kicked rotator with $K_{0}=2.5, L=1$, and $\varepsilon=10^{-3}$ (full curve). The support of the initial (characteristic) density is $(q, p) \in[0,0.2] \times[0,0.2]$. The dotted curve gives the exponential decay determined by the Lyapunov coefficient (about 0.534 ), while the dashed line shows the $\propto t^{-0.55}$ behavior. The decay of correlator $D$ for the same initial density and for twice the time $t$ is also shown (dot-dashed curve.)

(4) with $\left.F_{0}=K_{0} \sin \theta\right)$ in the regime with mixed phase space dynamics ( $\alpha \approx 0.55$ for $K_{0}=2.5, L=1$, see Fig. 4 ).

Finally we remark that the short time Lyapunov decay of fidelity is by no means a typical feature of correlation functions. This can be clearly seen in the dot-dashed curve of Fig. 4 , which represents the decay of the correlator $D(t)=(C(t)-$ $C(\infty)) /(C(0)-C(\infty))$, with $C(t)=\int_{\Omega} \mathrm{d} \vec{x} \rho_{0}(\vec{x}, t) \rho(\vec{x}, 0)$. Actually the short time decay of $D(t)$ is determined by the motion of the "center of mass" of the initial distribution $\rho(\vec{x}, 0)$, a trivial effect which is suppressed in fidelity due to the backward evolution.

In conclusion, in chaotic systems the asymptotic decay of classical fidelity, exponential or power law, is analogous to the asymptotic decay of correlation functions. It would be interesting to understand what are the implications of such connection for the decay of quantum fidelity.

\section{STABILITY OF QUANTUM MOTION UNDER SYSTEM'S PERTURBATIONS}

In this section we discuss the same question as in the previous one, now in the light of quantum mechanics, namely the stability of quantum motion against system's perturbation. We define the quantum fidelity in analogy to (1) as

$$
f(t)=\left|\left\langle\psi_{\varepsilon}(t) \mid \psi_{0}(t)\right\rangle\right|^{2}
$$

where

$$
\left|\psi_{\varepsilon}(t)\right\rangle=U_{\varepsilon}^{t}|\psi\rangle, \quad\left|\psi_{0}(t)\right\rangle=U_{0}^{t}|\psi\rangle
$$

are perturbed and unperturbed propagators, respectively, generated by the Hamiltonian $H_{\varepsilon}=H_{0}+\varepsilon V$, namely $U_{\varepsilon}^{t}=$ $\exp \left(-\mathrm{i} H_{\varepsilon} t / \hbar\right)$.

As it is discussed in more detail in contribution [27], the quantum fidelity (6) is expected to follow the classical fidelity
(1) up to the Ehrenfest time, which for a chaotic system with effective Lyapunov exponent $\lambda$, reads as $t^{*}=\ln (1 / \hbar) /(2 \lambda)$.

Obviously, for times shorter than $t^{*}$, the decay of quantum fidelity is determined by classical mechanics. For initial localized wave-packets $|\psi\rangle$ we expect initial exponential decay of fidelity with perturbation independent (Lyapunov) exponent $\lambda$

$$
f_{\text {Lyap }}(t)=\exp (-\lambda t) \text {. }
$$

For sufficiently strong perturbation strength $\varepsilon$, namely $\sigma:=$ $\varepsilon / \hbar \gg 1$, the quantum fidelity drops to a saturation value $f(\infty) \sim 1 / N$ (where $N$ is the dimension of the Hilbert space, $N \sim \hbar^{-d}$ and $d$ is the number of degrees of freedom) before the Ehrenfest time $t^{*}$ is reached. This regime is usually referred to as the Lyapunov regime of fidelity decay and has been first described in Ref. [10].

When the dimensionless parameter $\sigma$ becomes less than one, $\sigma<1$, then one may start to use time-dependentperturbation theory in order to calculate the fidelity decay. This regime is usually referred to as the Fermi-Golden-Rule regime, and in the case of classically chaotic (mixing) dynamics, fidelity decays exponentially

$$
f_{\mathrm{FGR}}(t)=\exp (-\Gamma t) .
$$

The exponent $\Gamma$ which can be understood also as the width of the Local density of states [12], can be computed [7] in terms of a 2-point time-correlation function of the perturbation $C(t)=\langle V V(t)\rangle-\langle V\rangle^{2}, V(t)=\exp \left(\mathrm{i} H_{0} t / \hbar\right) V \exp \left(-\mathrm{i} H_{0} t / \hbar\right)$, namely as

$$
\Gamma=\varepsilon^{2} D, \quad D:=\int_{-\infty}^{\infty} \mathrm{d} t C(t) .
$$

In fact, for sufficiently small effective Planck constant $\hbar$ the correlation function $C(t)$ and diffusion constant $D$ can be computed in terms of classical mechanics. One should note that $\sigma=1$ represents a border between classical $\sigma>1$, and quantum $\sigma<1$ behavior of fidelity [17].

However, this formula (9) works only for times shorter than the Heisenberg time $t_{\mathrm{H}}=2 \pi \hbar \rho$ where $\rho$ is the density of states. For longer times, quantum correlation function $C(t)$ starts to be dominated by quantum fluctuations and another approach has to be used. If the perturbation $\varepsilon$ is so small that a significant decay of fidelity is taking place after the Heisenberg time $t_{\mathrm{H}}$, then the stationary perturbation theory may be used [13] in order to derive a Gaussian decay of fidelity

$$
f_{\text {pert }}(t)=\exp \left(-4 \varepsilon^{2} D t^{2} / \hbar^{2}\right)
$$

Comparing the inverse decay rate $1 / \Gamma$ of the formula (9) with the Heisenberg time, we obtain the perturbative border [7, 12, 13] $\varepsilon_{\text {pert }} \sim \hbar^{d / 2+1}$ or $\sigma_{\text {pert }} \sim \hbar^{d / 2}$ such that for $\sigma<\sigma_{\text {pert }}$ the formula (11) is globally valid.

These three universal laws of decay of quantum fidelity (separated by two borders) have been derived for fully chaotic classical dynamics. However, a correlation-function approach [7], which can be used to derive Fermi-Golden-rule decay (9), can be used more generally, for example also for integrable or quasi-integrable classical dynamics. There, the absence of 


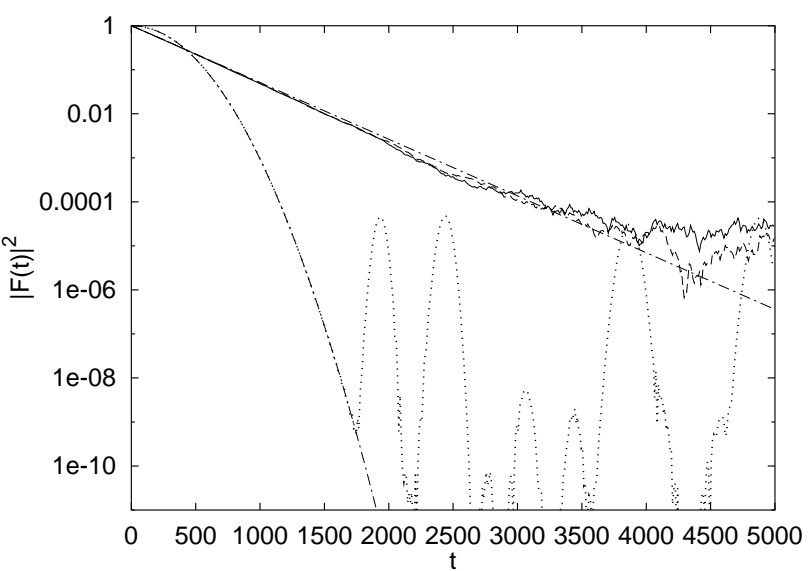

FIG. 5: Fidelity decay for two coupled kicked tops, for perturbation strength $\varepsilon=8 \cdot 10^{-4}$ and for angular momentum $J=200$. The upper curves are for $k=20$ (classically chaotic regime), solid curve for a coherent initial state and dashed curve for a random initial state, and the lower - dotted curve is for $k=1$ (KAM, quasi-regular regime) with a coherent initial state. The exponential and Gaussian chain curves give, respectively, the expected theoretical decays described by formulae (9) and (12).

decay of dynamical correlations, in a generic (regular) case yields quadratic decay of fidelity in the regime of linear response, $F(t)=1-C \varepsilon^{2} t^{2} / \hbar+O\left(\varepsilon^{4}\right)$. For initial Gaussian wave-packets one can even show that the global decay in such a case is a simple Gaussian

$$
f_{\text {regular }}(t)=\exp \left(-\varepsilon^{2} C t^{2} / \hbar\right)
$$

where the constant $C$ can be computed solely from the classical data, such as the classical limit of the perturbation observable $V$ and the parameters of initial wave-packet.

Comparing the quantum fidelity decays for chaotic (9) as compared to regular (12) classical mechanics, one finds that the former decays on a time scale $t_{\mathrm{ch}} \sim \hbar^{2} \varepsilon^{-2}$ whereas the latter decays on a time scale $t_{\text {reg }} \sim \hbar^{1 / 2} \varepsilon^{-1}$. Therefore, for sufficiently small perturbation $\varepsilon$ (for $\sigma \ll \hbar^{1 / 2}$ ) the asymptotic fidelity decay for classically chaotic dynamics is slower than for classically regular dynamics. (Note that for random initial states such a paradoxical behavior takes place even for $\sigma \ll 1$.) This behavior is not in contrast with the correspondence principle as it takes place for time scales beyond the breaking time $t^{*}$ for quantum classical correspondence. One should always keep in mind the non-commutativity of the limits $\hbar \rightarrow 0$ and $\varepsilon \rightarrow 0$.

Let us make a short illustration in terms of a simple numerical experiment. We will consider a system with two degrees of freedom, a pair of coupled kicked tops, described by two independent SU(2) variables (angular momenta) $\vec{J}_{1}$ and $\vec{J}_{2}$.

A quantum unitary propagator, with some external coupling parameter $k$, for one-period of the kick reads

$$
U=e^{-\mathrm{i} \frac{\pi}{2} J_{1 \mathrm{y}}} e^{-\mathrm{i} \frac{\pi}{2} J_{2 y}} e^{-\mathrm{i} k J_{1 \mathrm{z}} J_{2 \mathrm{z}} / J} .
$$

The perturbed propagator is obtained by perturbing the parameter $k$, so that $U_{\varepsilon}=U(k+\varepsilon)$. The generator of perturbation is therefore

$$
V=\frac{1}{J^{2}} J_{1 \mathrm{z}} J_{2 \mathrm{z}}
$$

The modulus of angular momentum $J$ is fixed and equal for both tops, and determines the effective value of Planck constant $\hbar=1 / J$. The total Hilbert space dimension is $N=$ $1 /(2 J+1)^{2}$.

We have chosen two regimes of qualitatively different classical dynamics of the system, namely non-ergodic (KAM) regime for $k=1$ where the vast majority of classical orbits are stable, and the mixing regime for $k=20$ where no significant traces of stable classical orbits. As for initial states we take direct products of $\mathrm{SU}(2)$ coherent states centered at two points $\left(\vartheta_{1,2}, \varphi_{1,2}\right)$ on the two spheres. In Fig. 5 we show the fidelity decay at $J=200$ and $\varepsilon=8 \cdot 10^{-4}$ in nonergodic and mixing cases started from the coherent state with $\left(\vartheta_{1}, \varphi_{1}\right)=\left(\vartheta_{2}, \varphi_{2}\right)=\pi(1 / \sqrt{3}, 1 / \sqrt{2})$. In the most important quantum regime, where $t^{*} \ll t \ll t_{\mathrm{H}}$, we find excellent agreement between the theoretical predictions (9) and (12) and the numerics. In the ergodic-mixing regime $(k=20)$ we show for comparison also the fidelity decay for a random initial state which is (due to ergodicity) almost identical to the case of coherent initial state.

In this section we have shown that the behavior of quantum fidelity is, beyond the Ehrenfest time scale $t^{*}$, drastically different that for a classical fidelity. In general we may claim that quantum fidelity decays slower than the classical fidelity. Recently, we have discovered even more drastic particular situation, namely the phenomenon of quantum freeze of fidelity [28] which takes place for perturbations wit all diagonal elements identically vanishing in the eigenbasis of the unperturbed Hamiltonian. Such cases can sometimes emerge naturally due to symmetry.

\section{CHAOS INDUCED DECOHERENCE}

In the previous sections we have discussed the stability of quantum motion in the light of the corresponding classical motion. In this connection, the question whether, in order to have the quantum to classical transition, external noise (or coupling to external macroscopic number of degrees of freedom) is necessary or not, remains unclear. Indeed it is generally accepted that external noise may induce the non-unitary evolution leading to the decay of non-diagonal matrix elements of the density matrix in the eigenbasis of the physical observables, thus restoring the classical behavior. On the other hand it has also been surmised that external noise, being sufficient, is not necessary. A new type of decoherence - the dynamical decoherence - has been proposed [5], without any noise and only due to the intrinsic chaotic evolution of a pure quantum state. The simplest manifestations of dynamical decoherence are the fluctuations in the quantum steady state which, in the quasi-classical region, is a superposition of very many eigenfunctions. In case of a quantum chaotic - ergodic steady state - all eigenfunctions essentially contribute to the fluctuations and their contribution is statistically independent 


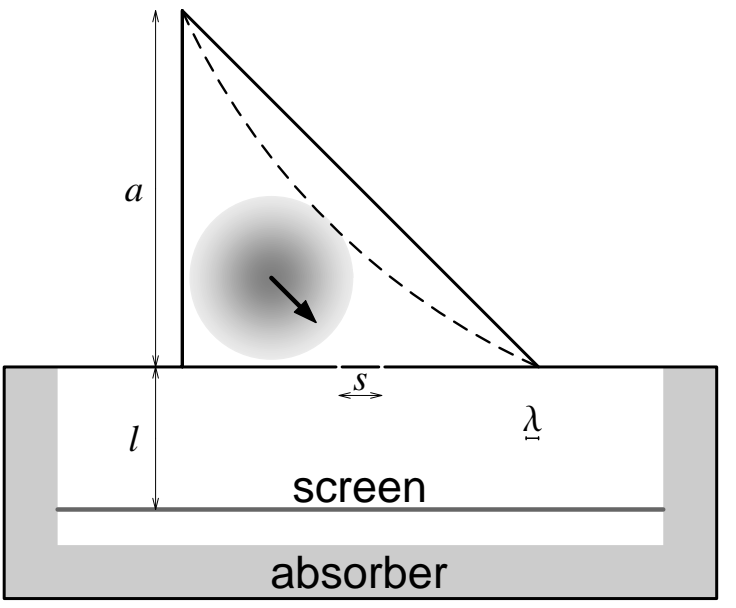

FIG. 6: The geometry of the numerical double-slit experiment. All scales are in proper proportions. The two slits are placed at a distance $s$ on the lower side of the billiard

[5]. This fact suggests the complete quantum decoherence in the final steady state for any initial state even though the steady state is formally a pure quantum state. Yet this argument is not completely convincing and a more clear evidence is required. In a recent paper [29] this question has been discussed by considering one of the basic experiments on which rests quantum mechanics, namely a phenomenon which, in the words of Richard Feynmann [30], "... is impossible absolutely impossible, to explain in any classical way, and which has in it the heart of quantum mechanics. In reality, it contains the only mystery." : the double slit experiment.

The following numerical, double-slit experiment has been performed. The time dependent Schrödinger equation $\mathrm{i} \hbar \frac{\partial}{\partial t} \Psi(x, y, t)=\hat{H} \Psi(x, y, t)$, with $\hat{H}=\hat{p}^{2} /(2 m)$, has been solved numerically [31] for a quantum particle which moves freely inside the two-dimensional domain as indicated in Fig. 6 (full line). Note that the domain is composed of two regions which are connected only through two narrow slits. We refer to the upper bounded region as to the billiard domain, and to the lower one as the radiating region. The scaled units have been used in which Planck's constant $\hbar=1$, mass $m=1$, and the base of the triangular billiard has length $a=1$. The initial state $\Psi(t=0)$ is a Gaussian wave packet (coherent state) centered at a distance $a / 4$ from the lower-left corner of the billiard (in both Cartesian directions) and with velocity $\vec{v}$ pointing to the middle between the slits. The screen is at a distance $l=0.4$ from the base of the triangle. The magnitude of velocity $v$ (in our units equal to the wave-number $k=v$ ) sets the de Broglie wavelength $\lambda=2 \pi / k$. In our experiment we have chosen $k=180$ corresponding to approximately 1600 th excited states of the closed quantum billiard. The slits distance has been set to $s=0.1 \approx 3 \lambda$ and the width of the slits is $d=\lambda / 4$. The wave-packet is also characterized by the position uncertainty $\sigma_{x}=\sigma_{y}=0.24$. This was chosen as large as possible in the present geometry in order to have a small uncertainty in momentum $\sigma_{k}=1 /\left(2 \sigma_{x}\right)$.

The lower, radiating region, should in principle be infinite. Thus, in order to efficiently damp waves at finite boundaries, we have introduced an absorbing layer around the radiating region. More precisely, in the region referred to as absorber, we have added a negative imaginary potential to the Hamiltonian $H \rightarrow H-\mathrm{i} V(x, y), V \geq 0$, which, according to the time dependent Schrödinger equation, ensures exponential damping in time. In order to minimize any possible reflections from the border of the absorber, we have chosen $V$ to be smooth, starting from zero and then growing quadratically inside the absorber. No significant reflection from the absorber was detected and this ensures that the results of our experiment are the same as would be for an infinite radiating region.

While the wave-function evolves with time, a small probability current leaks from the billiard and radiates through the slits. The radiating probability is recorded on a horizontal line $y=-l$ referred to as the screen. The experiment stops when the probability that the particle remains in the billiard region becomes vanishingly small. We define the intensity at the position $x$ on the screen as the perpendicular component of the probability current, integrated in time

$$
I(x)=\left.\int_{0}^{\infty} \mathrm{d} t \operatorname{Im} \Psi^{*}(x, y, t) \frac{\partial}{\partial y} \Psi(x, y, t)\right|_{y=-l} .
$$

By conservation of probability the intensity is normalized, $\int_{-\infty}^{\infty} \mathrm{d} x I(x)=1$, and is positive $I(x) \geq 0 . I(x)$ is interpreted as the probability density for a particle to arrive at the screen position $x$. According to the usual double slit experiment with plane waves, the intensity $I(x)$ should display interference fringes when both slits are open, and would be a simple unimodal distribution when only a single slit is open. This is what we wanted to test with a more realistic, confined geometry. The resulting intensities are shown in Figs. 7, 8(red curves).

Indeed, a very clear (symmetric) interference pattern was found, with a visibility of the fringes depending on the parameters of the initial wave-packet. This can be heuristically understood as a result of integrability of the corresponding billiard dynamics. Namely, the classical ray dynamics inside a $\pi / 4$ right triangular billiard is regular representing a completely integrable system. We know that each orbit of an integrable system is characterized by the fact that, since the classical motion in $2 \mathrm{~N}$ dimensional phase space is confined onto an $N$ invariant torus, at each point in position space, e.g. at the positions of the slits, only a finite number of different momenta (directions) are possible. Thus the quantum wavefunction, in the semiclassical regime, is expected to be locally a superposition of finitely many plane-waves [32] and the interference pattern on the screen is expected to be simply a superposition of fringes using these plane waves. In our case of an integrable $\pi / 4$ right triangular billiard, different directions result from specular reflections with the walls. In contrast to the idealized plane-wave experiment in infinite domain where interference pattern depends on the direction of the impact, the fringes here were always symmetric around the center of the screen. This is a consequence of the presence of the vertical billiard wall, namely due to collisions with this wall each im- 


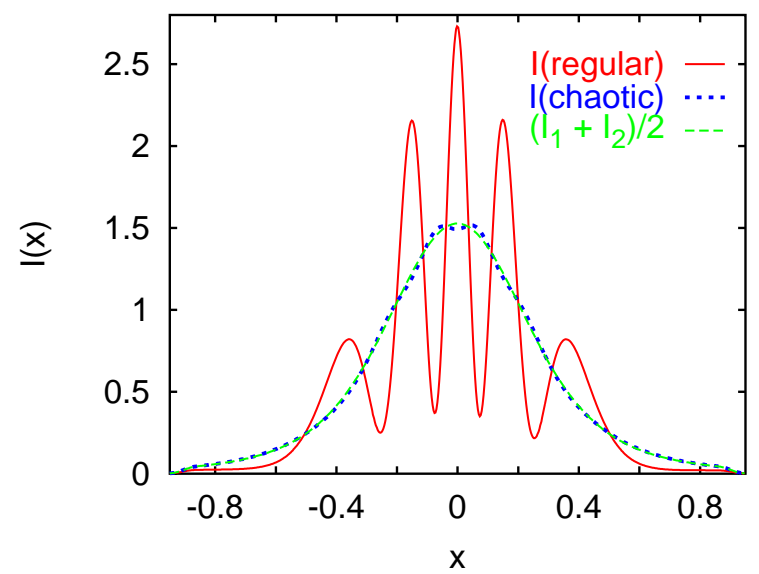

FIG. 7: The total intensity after the double-slit experiment as a function of the position on the screen. $I(x)$ is obtained as the perpendicular component of the probability current, integrated in time. The red full curve indicates the case of regular billiard, while the blue dotted curve indicates the case of chaotic one. The green dashed curve indicates the averaged intensity over two 1-slit experiments, with either the regular or chaotic billiard (with results being practically the same, see Fig. 8).

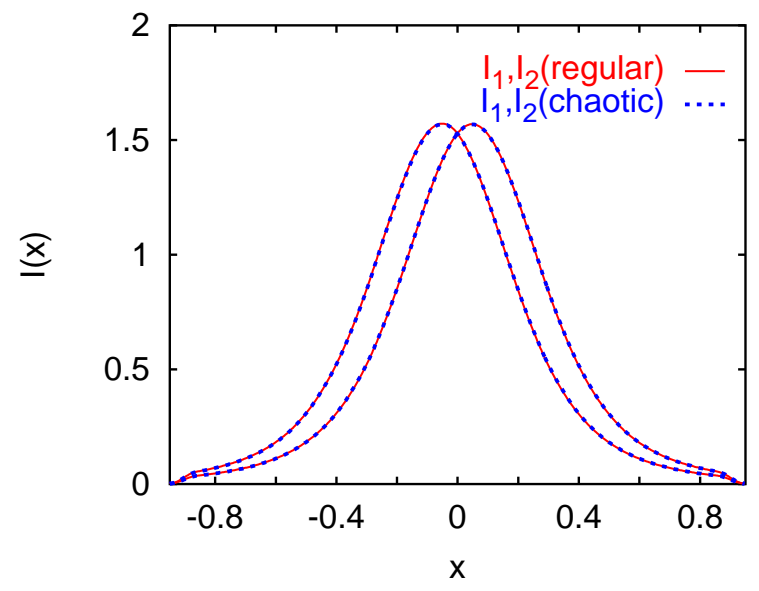

FIG. 8: The two pairs of curves represent the intensities on the screen for the two 1-slit experiments (with either one of the two slits closed). The red full curves indicate the case of the regular billiard while the blue dotted ones indicate the case of chaotic billiard.

pact direction $\left(v_{x}, v_{y}\right)$ is always accompanied with a reflected direction $\left(-v_{x}, v_{y}\right)$. The pattern on the screen is then a symmetric superposition of the two interference images, one being a reflection $(x \rightarrow-x)$ of the other. In this way one can also understand that the visibility of the interference fringes may vary with the direction of the initial packet.

We also remark that the spacing between interference fringes is in agreement with the usual condition for plane waves that the difference of the distances from the two slits to a given point on the screen is an integer multiple of $\lambda$.

Let us now make a simple modification of our experiment. We replace the hypotenuse of the triangle by the circular
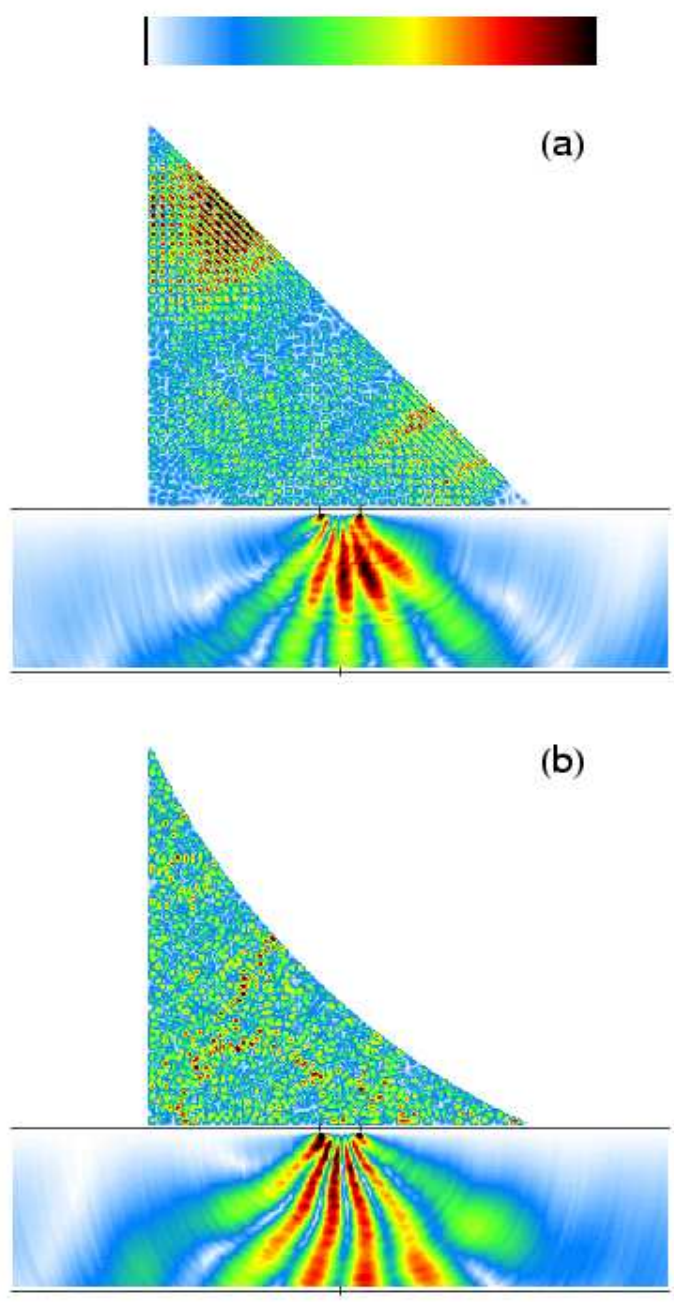

FIG. 9: Typical snapshots of the wave-function (plotted is the probability density) for the two cases: (a) for the regular billiard at $t=0.325$, and (b) for the chaotic billiard at $t=0.275$ (both cases correspond to about half the Heisenberg time). The probability density is normalized separately in both parts of each plot, namely the probability density, in absolute units, in the radiating region is typically less than $1 \%$ of the probability density in the billiard domain. The screen, its center, and the positions of the slits are indicated with thin black lines. Please note that the color code on the top of the figure is proportional to the square root of probability density.

arc of radius $R=2$ (dashed curve in Fig. 6). This change has a dramatic consequence for the classical ray dynamics inside the billiard, namely the latter becomes fully chaotic. In fact such a dispersive classical billiard is rigorously known to be a $K$-system [3]. Quite surprisingly, this has also a dramatic effect on the result of the double slit experiment. The interference fringes almost completely disappear, and the intensity can be very accurately reproduced by the sum of intensities $\left(I_{1}(x)+I_{2}(x)\right) / 2$ for the two experiments where only a single slit is open. This means that the result of such experiment is the same as would be in terms of classical ray dynamics. Notice however, that at any given instant 
of time, there is a well defined phase relation between the wave function at both slits. Yet, as time proceeds, this phase relation changes, and it is lost after averaging over time. This is nicely illustrated by the snapshots of the wave-functions in the regular and chaotic case shown in Fig. 9. While in the regular case, the jets of probability emerging from the slits always point in the same direction and produce a clear time-integrated fringe structure on the screen, in the chaotic case, the jets are trembling and moving left and right, thus upon time-integration they produce no fringes on the screen [33].

The results of this numerical experiment can be understood in terms of fast decay of spatial correlations of eigenfunctions of chaotic systems. In the limit of small slits opening $d \ll \lambda$, the intensity on the screen, according to simple perturbation expansion in the small parameter $d / \lambda$, can be written as

$$
I(x)=I_{1}(x)+I_{2}(x)+C(s) f(x),
$$

where $f(x)$ is some oscillatory function determining the period of the fringes, and $C(s)$ is the spatial correlation function of the normal derivative of the eigenfunctions $\Psi_{n}$ of the closed billiard at the positions $(-s / 2,0)$ and $(s / 2,0)$ of the slits, written in the Cartesian frame with origin in the middle point between the slits. In particular, $C(s)=$ $\alpha \sum_{n}\left|c_{n}\right|^{2} \partial_{y} \Psi_{n}(-s / 2,0) \partial_{y} \Psi_{n}(s / 2,0)$, where $c_{n}$ are the expansion coefficients of the initial wave-packet in the eigenstates $\Psi_{n}$, and $\alpha$ is a constant such that $C(0)=1$. Note that this eigenstate correlation function $C(s)$, which also depends on the initial state through the expansion coefficients $c_{n}$, is directly proportional to the visibility of the fringes. One may use well known random plane wave model for chaotic billiards [32], in combination with a method of images to account for the boundary condition, to show that quantum chaotic eigenstates exhibit decaying correlations with $C(s)=J_{1}(k s) /(k s)$ where $J_{1}$ is a first order Bessel function, whereas for regular systems $C(s)$ typically does not decay (but oscillates) so it produces interference fringes. In our case of half-square billiard we find, for large $k, C(s)=e^{-\sigma_{k}^{2} s^{2} / 2}\left(k_{x}^{2} \cos \left(k_{y} s\right)+\right.$ $\left.k_{y}^{2} \cos \left(k_{x} s\right)\right) / k^{2}$. The Gaussian prefactor can easily be understood, namely there is no interference if the size of the wavepacket is smaller than the slit-distance, or equivalently, if uncertainty in momentum $\sigma_{k}$ is much larger than $1 / s$.

Disappearance of interference fringes can be directly related to decoherence. If $A$ is a binary observable $A \in$ $\{1,2\}$ which determines through which slit the particle went, then $C(s)$ is proportional to the non-diagonal matrix element $\langle 1|\rho| 2\rangle$ of the density matrix in the eigenbasis of $A$, and is thus a direct indicator of decoherence.

The result presented here provides therefore, from one hand, a vivid and fundamental illustration of the manifestations of classical chaos in quantum mechanics. On the other hand it shows that, by considering a pure quantum state, in absence of any external decoherence mechanism, internal dynamical chaos can provide the required randomization to ensure quantum to classical transition in the semiclassical region. The effect described in this letter should be observable in a real laboratory experiment.

\section{CONCLUSIONS}

In this paper we have presented two different, general illustrations of observable effects of quantum chaos. On one hand, we have shown that sensitivity to system's perturbations is clearly connected with the nature of the underlying classical dynamics. The concepts of classical and quantum Loschmidt echoes are relatively new but may have important implications in statistical physics and in the field of quantum information and quantum computation. On the other hand, we have shown that quantum chaos can act also as a source of noise thus producing effects equivalent to decoherence, such as destroying interference fringes in a double slit experiment.

The work has been financially supported in part by the grant DAAD19-02-1-0086, ARO United States, and by the grant (T.P.) P1-0044 of the Ministry of Science, Education and Sports of Republic of Slovenia.
[1] F. Haake, Quantum Signatures of Chaos, 2nd edition (SpringerVerlag, Heidelberg, 2001).

[2] H.-J. Stöckmann, Quantum Chaos - An Introduction (Cambridge University Press, Cambridge, 1999).

[3] I.P. Cornfeld, S.V. Fomin and Y.G. Sinai, Ergodic theory (Springer-Verlag, 1982).

[4] G. Casati, B. V. Chirikov, I. Guarneri and D. L. Shepelyansky, Phys. Rev. Lett. 56, 2437 (1986).

[5] G. Casati and B. V. Chirikov, in: "Quantum chaos: between order and disorder" (Cambridge University Press, Cambridge, England, 1995) p.3; G. Casati and B. V. Chirikov, Physica D86, 220 (1995); G. Casati and B. V. Chirikov, Phys. Rev. Lett. 75 , 350 (1995).

[6] A. Peres, Phys. Rev. A 30, 1610 (1984).

[7] T. Prosen and M. Žnidarič, J. Phys. A 35, 1455 (2002); T. Prosen, Phys. Rev. E 65, 036208 (2002).
[8] G. Benenti, G. Casati and G. Strini, Principles of Quantum Computation and Information, Vol. I: Basic concepts (World Scientific, Singapore, 2004).

[9] M.A. Nielsen and I.L. Chuang, Quantum Computation and Quantum Information (Cambridge University Press, Cambridge, 2000).

[10] R.A. Jalabert and H.M. Pastawski, Phys. Rev. Lett, 86, 2490 (2001).

[11] F.M. Cucchietti, H.M. Pastawski and D.A. Wisniacki, Phys. Rev. E 65, 045206(R) (2002).

[12] Ph. Jacquod, P.G. Silvestrov and C.W.J. Beenakker, Phys. Rev. E 64, 055203(R) (2001),

[13] N.R. Cerruti and S. Tomsovic, Phys. Rev. Lett. 88, 054103 (2002).

[14] V.V. Flambaum and F.M. Izrailev, Phys. Rev. E 64, 036220 (2001). 
[15] Z.P. Karkuszewski, C. Jarzynski and W.H. Zurek, quant$\mathrm{ph} / 0111002$.

[16] D.A. Wisniacki and D. Cohen, quant-ph/0111125.

[17] G. Benenti and G. Casati, Phys. Rev. E 65, 066205 (2002).

[18] J. Vanicek and E. J. Heller, Phys. Rev. E 68, 056208 (2003).

[19] G. Benenti, G. Casati and G. Veble, Phys. Rev. E 67, 055202(R) (2003).

[20] B. Eckhardt, J. Phys. A: Math. Gen. 36, 371 (2003).

[21] G. Veble and T. Prosen, Phys.Rev.Lett. 92, 034101 (2004).

[22] D. Ruelle, Phys. Rev. Lett. 56, 405 (1986).

[23] J. Weber, F. Haake and P. Šeba, Phys. Rev. Lett. 85, 3620 (2000).

[24] M. Khodas, S. Fishman and O. Agam, Phys. Rev. E 62, 4769 (2000).

[25] F. Vivaldi, G. Casati and I. Guarneri, Phys. Rev. Lett. 51, 727 (1983).

[26] C. F. F. Karney, Physica 8D, 360 (1983); B.V. Chirikov and D.L. Shepelyansky, Physica 13D, 395 (1984).

[27] T. Prosen and M. Žnidarič, Braz. J. Phys. (2005), this volume.

[28] T. Prosen and M. Žnidarič, New J. of Phys. 109 (2003); Phys. Rev. Lett. (2005), in press.

[29] G. Casati and T. Prosen, "Quantum chaos and the double-slit experiment", nlin.CD/0403038.

[30] R. P. Feynman, Lecture Notes in Physics, Vol. 3 (Addison-
Wesley, 1965), p.1-1.

[31] We have implemented an explicit finite difference numerical method with $\lambda / h \approx 12$ mesh points per de Broglie wavelength $\lambda$ where $h$ is the step-size of the spatial discretization. The stability of the method was enforced by using unitary power-law expansion of the propagator, namely $\Psi(t+\tau)=$ $\sum_{j=0}^{n} \frac{1}{n !}\left(-\frac{\mathrm{i} \tau}{\hbar} \hat{H}\right)^{j} \Psi(t)$ where $\hat{H}=-\frac{\hbar^{2}}{2 m} \Delta$ and $\Delta$ is a discrete Laplacian. Using temporal step-size $\tau=h^{2}$, the required order $n$ to obtain numerical convergence within machine precision was typically small, $n<10$. The implementation of the finite difference scheme was straightforward for the triangular geometry, since the boundary conditions conform nicely to the discretized Cartesian grid. For the case of chaotic billiard, we used a unique smooth transformation $(x, y) \rightarrow(x, f(y))$ which maps the chaotic billiard geometry to the regular one, and slightly modifies the calculation of the discrete Laplacian without altering its accuracy (due to smoothness of $f(y)$ ).

[32] M. V. Berry, J. Phys. A: Math. Gen. 10, 2083 (1977).

[33] This is somewhat similar to what happens to a Wigner function of a quantum chaotic state - see: M. Horvat and T. Prosen, Wigner function statistics in classically chaotic systems, J. Phys. A: Math. Gen. 36, 4015 (2003) - when one projects it in the position space and obtains a smooth probability density. 